\title{
Antineutrophil cytoplasmic antibodies in sera from colectomised ulcerative colitis patients and its relation to the presence of pouchitis
}

M Esteve, J Mallolas, J Klaassen, A Abad-Lacruz, F González-Huix, E Cabré, F Fernández-Bañares, X Bertrán, E Condom, J Martí-Ragué, M A Gassull

\section{Department of Gastroenterology, Hospital Universitari 'Germans Trias i Pujol', Badalona, Catalonia, Spain M Esteve $\mathrm{J}$ Mallolas J Klaassen E Cabré \\ F Fernández-Bañares X Bertrán \\ M A Gassull}

Department of Gastroenterology, Hospital de Sant Llorenc, Viladecans, Catalonia, Spain A Abad-Lacruz

Department of Gastroenterology, Hospital 'Josep Trueta', Girona, Catalonia, Spain F González-Huix

Department of Pathology, Ciutat Sanitaria Universitaria de Bellvitge, L'Hospitalet, Catalonia, Spain E Condom

Department of Surgery, Ciutat Sanitaria Universitaria de Bellvitge, L'Hospitalet, Catalonia, Spain J Martí-Ragué

Correspondence to: Correspondence to: Universitari 'Germans Trias Universitari 'Germans Trias Pujol', Carretera del Canyet s/n, 08916 Badalona, Spain. Accepted for publication 13 December 1995

\begin{abstract}
Background-Few studies have evaluated the influence of colectomy on antineutrophil cytoplasmic antibody (ANCA) positivity in ulcerative colitis (UC). In small series of patients it has been suggested that ANCA positivity in UC might be predictive for development of pouchitis after colectomy.

Aims-To assess the prevalence of ANCA in UC patients treated by colectomy and a Brooke's ileostomy (UC-BI) or ileal pouch anal anastomosis (UC-IPAA), and the relation between the presence of ANCA, the type of surgery, and the presence of pouchitis.

Subjects- 63 UC patients treated by colectomy (32 with UC-BI and 31 with UCIPAA), 54 UC, and 24 controls.

Methods-Samples were obtained at least two years after colectomy. ANCA were detected by indirect immunofluorescent assay.

Results-There were no differences between patients with $(36 \cdot 3 \%)$ or without pouchitis $(35.0 \%)$ and between patients with UC (55\%), UC-BI (40.6\%), and UCIPAA $(35.4 \%)$. However, ANCA prevalence significantly decreases in the whole group of operated patients (38.0\%) compared with non-operated UC $(p=0 \cdot 044)$. Conclusions-The prevalence of ANCA in operated patients was significantly lower than in non-operated UC, suggesting that it might be related either to the presence of inflamed or diseased tissue. ANCA persistence is not related to the surgical procedure and it should not be used as a marker for predicting the development of pouchitis.

(Gut 1996; 38: 894-898)
\end{abstract}

Keywords: ulcerative colitis, proctocolectomy, ileal pouch anal anastomosis, antineutrophil cytoplasmic antibodies, pouchitis.

Ulcerative colitis (UC) and associated conditions such as primary sclerosing cholangitis are some of the diseases in which antineutrophil cytoplasmic antibodies (ANCA) have been described. ${ }^{1-3}$ UC associated ANCA (UCANCA) most commonly show an immunofluorescence perinuclear pattern. Although not universally accepted, ${ }^{3}$ cytoplasmic and mixed ANCA patterns have been described associated to UC. ${ }^{4-8}$ On the other hand, because the antigenic specificity of UC-ANCA is unknown, ${ }^{13910}$ they are referred to as atypical ANCA or X-ANCA by some authors. ${ }^{1-3}$

Few studies have specifically evaluated the influence of colectomy on ANCA positivity in UC. ${ }^{411-14}$ As well as only dealing with a small series of patients, none of them includes a large number of cases with Brooke's ileostomy, which are the only ones that may be considered absolutely free of disease. On the other hand, all these series report a high prevalence of ANCA in patients with pouchitis $(80-100 \%) .^{4711-13}$ Based on the results of these studies it has been suggested that ANCA assessment in UC may be useful in predicting the development of pouchitis in patients undergoing IPAA. ${ }^{2}$

The aims of this study were (a) to assess the prevalence of ANCA in UC patients treated with colectomy and either a Brooke's ileostomy or ileal pouch anal anastomosis, and (b) to determine the relation between the presence of ANCA, the type of surgery performed, and the presence of pouchitis.

\section{Methods}

\section{PATIENTS AND CONTROLS}

Ulcerative colitis patients treated with colectomy

One hundred and forty eight patients with a diagnosis of UC have had a colectomy since 1973 in three of four hospitals taking part in the study. The diagnosis had been established by the Lennard-Jones clinicopathological criteria. ${ }^{15}$ Patients with a history of indeterminate colitis were excluded from the study. Based on the information available, 92 of 114 patients living in Catalonia ( $31895 \mathrm{~km}^{2}$ ) could be contacted by phone and asked to participate in the study. Sixty three of them agreed to be included. Thirty two had a Brooke's ileostomy (UC-BI) (15 male, 17 female; 43 years (range: 23-76)) and 31 had an ileal pouch anal anastomosis (UC-ANCA) (18 male, 13 female; 34 years (range: 24-61)). The technique of IPAA included mucosectomy of the anorectal stump in five patients, whereas a staple ileal pouch anal anastomosis was performed in the remaining cases. In all patients a two loop J reservoir was made.

\section{Disease controls}

Fifty four UC patients who had not had an operation ( 35 male, 19 female; 40 years (range: 
TABLE I Clinical characteristics of the ulcerative colitis patients

\begin{tabular}{lc}
\hline & Patients \\
\hline Activity & \\
Inactive & 21 \\
Mild & 21 \\
Moderate & 11 \\
Severe & 1 \\
Extent & \\
Proctitis & 16 \\
Left sided colitis & 28 \\
Pancolitis & 10 \\
Extraintestinal manifestations & 5 \\
Treatment & \\
No treatment & $12^{\star}$ \\
Salicylates & 37 \\
Corticosteroids & 8 \\
Azathioprine & 7 \\
Corticosteroid refractory & 13 \\
\end{tabular}

^First episode. Time elapsed from diagnosis: 4 months (0-25).

19-74)), living in Catalonia, attending the 'Hospital Universitari Germans Trias i Pujol' were included in the study. The diagnostic criteria were the same as above.

Healthy controls

Twenty four volunteers, living in the same geographical area ( 11 male, 13 female; 43 years (range: 23-72)) acted as a healthy controls.

\section{CLINICAL ASSESSMENT}

In all patients a complete clinical assessment was performed at the time of blood sampling for ANCA detection. In non-operated UC patients this included: activity and extent of the disease, current treatment, time from diagnosis, extraintestinal manifestations, and outcome. The activity of the disease was assessed by means of the Truelove index. ${ }^{16}$ Table I shows the clinical characteristics of the non-operated UC patients. The assessment of UC patients treated with a colectomy included: time from diagnosis and surgery,

TABLE II Pouchitis disease activity index (PDAI) ${ }^{1415}$

\begin{tabular}{|c|c|}
\hline Criteria & Score \\
\hline \multicolumn{2}{|l|}{$\begin{array}{l}\text { Clinical } \\
\text { Stool frequency: }\end{array}$} \\
\hline $\begin{array}{l}\text { Usual postoperative stool frequency } \\
1-2 \text { Stools/day }>\text { postoperative usual } \\
3 \text { Or more stools/day }>\text { postoperative usual }\end{array}$ & $\begin{array}{l}0 \\
1 \\
2\end{array}$ \\
\hline Rectal bleeding: & \\
\hline $\begin{array}{l}\text { None or rare } \\
\text { Present daily }\end{array}$ & 0 \\
\hline \multicolumn{2}{|l|}{ Faecal urgency or abdominal cramps: } \\
\hline None & 0 \\
\hline Occasional & 1 \\
\hline Usual & 2 \\
\hline \multicolumn{2}{|l|}{ Fever (temperature $>37 \cdot 8^{\circ} \mathrm{C}$ : } \\
\hline Absent & 0 \\
\hline Present & 1 \\
\hline \multicolumn{2}{|l|}{ Endoscopic inflammation } \\
\hline Oedema & 1 \\
\hline Granularity & 1 \\
\hline Friability & 1 \\
\hline Loss of vascular pattern & 1 \\
\hline Mucous exudate & 1 \\
\hline Ulceration & 1 \\
\hline \multicolumn{2}{|l|}{$\begin{array}{l}\text { Acute histological inflammation } \\
\text { Polymorphonuclear leucocyte infiltration: }\end{array}$} \\
\hline Mild & 1 \\
\hline Moderate + crypt abscess & 2 \\
\hline Severe + crypt abscess & 3 \\
\hline \multicolumn{2}{|l|}{ Ulceration per low power field (mean): } \\
\hline $\begin{array}{l}<25 \% \\
25-50 \%\end{array}$ & $\begin{array}{l}1 \\
2\end{array}$ \\
\hline$>50 \%$ & 3 \\
\hline
\end{tabular}

extraintestinal manifestations, previous and current treatment, and postoperative outcome.

Eight patients with UC-BI were previous UC-IPAA that had failed. The primary reason for pouch excision was chronic pouchitis resistant to medical treatment in two patients and fistula and pelvic sepsis in six patients. Four of the second group also had chronic continuous pouchitis. None of these patients had indeterminate or Crohn's colitis. In UC-IPAA patients, the degree of pouch inflammation was assessed by means of the modified Moskowitz index ${ }^{17} 18$ (Table II). Pouchitis was defined as a score equal or greater than 7 . Eleven of 31 UC-IPAA patients fulfilled this criterion at the time of inclusion.

Previous history of confirmed pouchitis was also recorded. Four of 11 patients with pouchitis at the time of inclusion had history of chronic continuous pouchitis resistant to medical treatment. Table III shows clinical data of UC patients treated with a colectomy. Some UC-IPAA patients were taking more than one drug. None of the patients included had primary sclerosing cholangitis.

\section{ANTINEUTROPHIL ANTIBODIES DETECTION}

Serum was obtained of all patients and controls. In operated patients, samples were obtained at least two years after colectomy. ANCA were detected using an indirect immunofluorescence microscopy detection method. Commercially available slides containing cytospin smears of human ethanol fixed neutrophils (INOVA Diagnostic, San Diego, CA) were used as substrate. Diluted serum samples in phosphate buffered saline (PBS) were incubated on the slides for 30 minutes. After washing in PBS, the slides were incubated again for 30 minutes with fluorescein conjugated rabbit immunoglobulins to human immunoglobulin G (DAKO, Copenhagen, Denmark) at a predetermined optimal dilution of $1 / 100$ in PBS. Slides were washed in PBS twice, mounted in glycerol, and read immediately under fluorescence microscopy at $400 \times$ (Axioplan, Zeiss, Germany).

Slides were read by two independent observers who were unaware of the clinical diagnosis. Control ANCA positive and negative sera and PBS were included in each test batch. A serum was considered positive for ANCA when a clear perinuclear fluorescence at a 1:20 dilution was observed or when a

TABLE III Clinical characteristics of the UC patients treated with a colectomy

\begin{tabular}{|c|c|c|c|}
\hline & $U C-B I$ & $\begin{array}{l}\text { UC-IPAA } \\
\text { pouchitis }\end{array}$ & $\begin{array}{l}\text { UC-IPAA } \\
\text { no pouchitis }\end{array}$ \\
\hline Years from diagnosis & $14(4-36)$ & $6(2-34)$ & $7(2-24)$ \\
\hline Years from surgery & $9(2-16)$ & $4(2-9)$ & $4(2-9)$ \\
\hline Clinical score ${ }^{\star}$ & - & $2(0-4)$ & $0(0-3)$ \\
\hline Endoscopic score* & - & $4(3-6)$ & $0(0-3)$ \\
\hline Histological score ${ }^{\star}$ & - & $2(1-5)$ & $1(0-3)$ \\
\hline Global score* & - & $8(7-11)$ & $3(1-6)$ \\
\hline \multicolumn{4}{|l|}{ Treatment (patients): } \\
\hline No treatment & 32 & 3 & 14 \\
\hline Salicylates & 0 & 2 & 3 \\
\hline Local corticosteroids & 0 & 3 & 0 \\
\hline Antibioticst & 0 & 4 & 5 \\
\hline
\end{tabular}

^Pouchitis disease activity index; tmetronidazole/tetracyclines. 
cytoplasmic staining was observed at a 1:40 dilution. The second cut off dilution was chosen to eliminate unspecific cytoplasmic positivity yielded by some negative sera. All negative sera and those given as positive by only one observer were retested blindly. Serum samples given as positive by only one observer in duplicate assay were considered as negative. Positive ANCA samples were titrated to a dilution of $1 / 1280$.

To differentiate positive ANCA from antinuclear antibodies (ANA) that mimic ANCA, all positive sera were tested with $\mathrm{HEp}-2$ cells and rat liver sections, which are highly sensitive for ANA detection. All ANA positive sera with stronger titres than that observed in neutrophil slides were considered as ANCA negative. This occurred in four patients (three nonoperated and one operated) and one healthy control.

All assays were performed at the Research Laboratories of the Hospital Universitary 'Germans Trias i Pujol'.

\section{STATISTICAL ANALYSIS}

Comparison of the frequencies between patients with or without pouchitis were analysed using the two tail $\chi^{2}$ and Yates's correction. The frequencies between UC, UC-IB, and UC-IPAA were compared using a two tail $\chi^{2}$ and the comparison between operated and non-operated UC patients was performed using a left sided Fisher exact test. Titres were compared using the Kruskal-Wallis test. The Spearman rank correlation coefficient was used to determine associations between titres and the time elapsed from surgery. The results are expressed by means of median and frequencies with their range and $95 \%$ confidence intervals (CI), respectively. The statistical procedures were performed using the programs of the Biomedical Data Processing, BMDP (BMDP, Statistical Software, Los Angeles, California, 1986).

ETHICAL CONSIDERATIONS

The study was performed in accordance with the 1975 Declaration of Helsinki ethical guidelines and was approved by the Research and Ethical Committees of the Hospitals.

Results

Table IV shows the number of ANCA positive patients and controls in the groups studied.

TABLE IV Number and percentage of $A N C A$ positive patients

\begin{tabular}{lrc}
\hline & Positive & Negative \\
\hline Ulcerative colitis & $30(55 \cdot 5 \%)^{\star}$ & 24 \\
UC-BI & $13(40 \cdot 6 \%) \dagger$ & 19 \\
UC-IPAA with pouchitis & $4(36 \cdot 3 \%) \ddagger$ & 7 \\
UC-IPAA without pouchitis & $7(35 \cdot 0 \%) \S$ & 13 \\
Healthy controls & 0 & 24 \\
\hline
\end{tabular}

UC-BI = total proctocolectomy plus Brookes ileostomy,

UC-IPAA=proctocolectomy plus ileal pouch anal anastomosis. UC-IPAA = proctocolectomy plus ileal pouch anal anastomosis. 26 m-ANCA; 1 c-ANCA; 3 mixed pattern, $\nmid 11$ p-ANCA; 2 mixed pattern,
The positivity of ANCA was significantly higher in all groups studied compared with the healthy control group $(\mathrm{p}<0 \cdot 0001)$.

\section{Relation between $A N C A$ positivity and the presence of pouchitis}

In patients with UC-IPAA, there were no differences in ANCA positivity between those with $(36.3 \%$ (CI: 10.9 to $69 \cdot 2)$ ) or without pouchitis $(35 \cdot 0 \%$ (CI: $15 \cdot 5$ to $59 \cdot 2)$ ) $(p=0.720)$.

Two of four (CI: $6 \cdot 7$ to $93 \cdot 2 \%$ ) patients with chronic continuous pouchitis and four of eight UC-IPAA failure (CI: $15 \cdot 7$ to $84 \cdot 3 \%$ ) were ANCA positive.

As the percentage of ANCA positive patients was the same as those with or without pouchitis, they were considered together and compared with both patients treated with a colectomy and non-operated UC patients to determine the influence of a Brooke's ileostomy upon ANCA positivity.

\section{Relation between ANCA positivity and the type of surgery performed}

There were no differences in ANCA positivity between patients with UC-BI $(40.6 \%$ (CI: $23 \cdot 7$ to $59 \cdot 3)$ ) and UC-IPAA (35.4\% (CI: $19 \cdot 2$ to $54 \cdot 6)$ ) and non-operated UC patients $(55 \%$ (CI: $41 \cdot 4$ to $69 \cdot 1))(p=0 \cdot 154)$.

Because there were no significant differences between both groups of operated patients, they were considered together (both UC-BI and UC-IPAA) and compared with UC nonoperated patients to determine if colectomy decreases ANCA positivity. There was a significant decrease in ANCA prevalence in operated patients $-38 \cdot 0 \%$ (CI: $26 \cdot 1$ to $51 \cdot 2)$ compared with non-operated UC (55\%) $(\mathrm{p}=0 \cdot 044)$.

The Figure shows the titres of ANCA and UC, UC-BI, and UC-IPAA. There were no differences in ANCA titres between groups

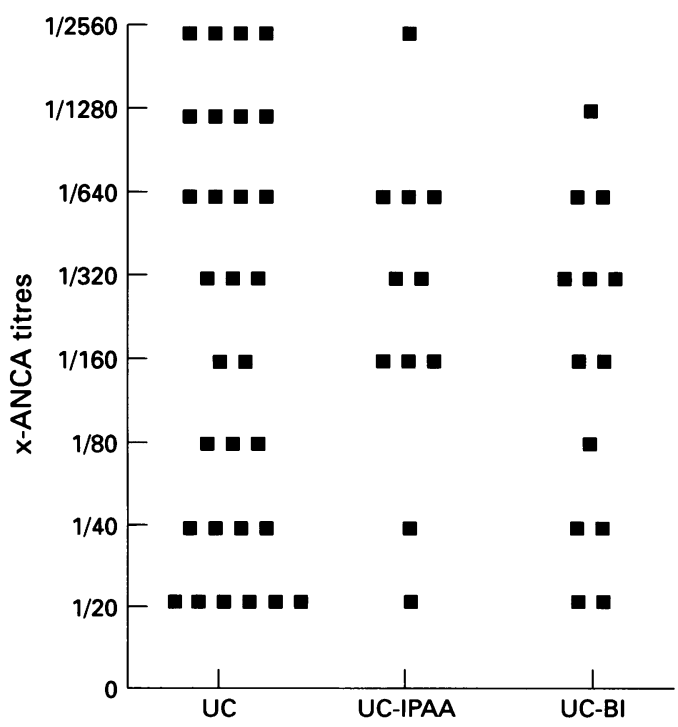

Titres of ANCA in non-operated ulcerative colitis patients (UC), patients treated with a colectomy plus ileal pouch anal anastomosis (UC-IPAA) and patients treated with a colectomy plus Brooke's ileostomy (UC-BI). 
$(p=0.755)$. There was also no correlation between the time elapsed from surgery and the titres of ANCA $(r=-0 \cdot 098)$.

\section{Discussion}

There is no agreement about those immunofluorescence ANCA patterns that should be considered associated with UC. Some laboratories have considered that all UC-ANCA are of the perinuclear type, ${ }^{919-21}$ whereas others also include those less frequent cases showing either diffuse cytoplasmic or mixed pattern. ${ }^{4-8}$ We share this second view as, until the specific antigen(s) are identified, it does not seem reasonable to exclude any type of ANCA pattern when an unequivocal immunofluorescence staining is found.

One of the aims of this study was to assess if removal of the colon results in a decline of ANCA in UC patients and if there is a relation to the type of surgery performed. The percentage of ANCA positivity in our UC non-operated patients $(55 \%)$ is similar to other reports, ${ }^{6} 2223$ and is within the range described in published reports $(40-80 \%) .4-619-25$ Whether or no circulating ANCA persist long term after colectomy in UC patients is not well established. Although most studies have reported the persistence of ANCA after surgery, ${ }^{4}$ 711-13 25 there are some reports of postoperative ANCA disappearance. ${ }^{14}{ }^{26}$ However, most studies include a small number of patients and none of them includes a large number of cases with Brooke's ileostomy.

Although there are no data about the time course of ANCA behaviour after colectomy, we thought that two years might be a reasonable period of time for assessing ANCA clearance, assuming that they are related to the presence of diseased tissue. We have not found a significant difference in ANCA positivity between non-operated UC patients, those with UC-BI, and those with UC-IPAA, showing that these antibodies persist in the serum in a high percentage of UC operated patients long term after colectomy. However, we found a significant decrease of ANCA positivity when both groups of operated patients were considered together (that is, increasing twofold the number of operated patients). This finding might disclose the existence of a weak relation between ANCA positivity and inflammation, as has been shown in some studies. ${ }^{6}{ }^{26}$ In fact, our non-operated patients showed a nonsignificant trend towards a greater percentage of ANCA positivity in those with active versus inactive UC $(63 \% v 43 \% ; p=0 \cdot 13)$, and in those with extensive disease versus proctitis $(63 \% v 31 \% ; p=0.07)$. An alternative explanation would be that ANCA clearance after colectomy was a very slow process taking more than two years and a prospective and paired assessment before and after surgery in 15 UC patients found a significant decrease in ANCA titres a mean of two years after colectomy. ${ }^{14}$ In our study, no differences between groups with respect to ANCA titres, or any relation between the titres of ANCA and the time elapsed from surgery were found. However, as this was not a paired comparison, interindividual differences in the ability of antibody production may have prevented the discovery of such differences.

In this series we found a similar frequency of ANCA positivity between those patients with and without pouchitis. In contrast, several studies have reported a higher prevalence of ANCA in patients with pouchitis. ${ }^{411-13}$ However, most of them include a small number of patients with pouchitis or it is not well defined, or both. ${ }^{41-13}$ In this regard, to distinguish between relapsing and chronic continuous pouchitis may be relevant. In the largest reported series, in which all patients with pouchitis had chronic inflammation resistant or dependent on medical treatment, frequency of ANCA positivity was $100 \%{ }^{7}$ The authors suggest that the strong relation between ANCA and pouchitis would be related to a specific disease pattern (chronic continuous pouchitis) rather than to the mere presence of inflammation. ${ }^{7}$ In the remaining studies, it is not stated if those patients with pouchitis had relapsing or chronic continuous disease. However, a recent study published as an abstract, showed that those patients without pouch inflammation at inclusion, but with previous episodes of pouchitis, had a $33.3 \%$ of ANCA positivity. ${ }^{12}$ This figure is similar to the percentage found in our patients with pouchitis, most of them having relapsing disease. On the other hand, in our series two of four chronic resistant pouchitis and four of eight IPAA failures had positive ANCA.

Taken as a whole, all these data suggest that ANCA would not predict the development of relapsing pouchitis, and the strong association between ANCA positivity and chronic continuous pouchitis deserves further confirmation. However, even if a $100 \%$ association (sensitivity) could be confirmed, the low specificity of ANCA for chronic continuous pouchitis $^{7}$ will make the use of this marker doubtful as a predictor of this condition.

Although all these queries could be only answered by means of a longitudinal study, its practicability seems to be questionable. Such a study would be very long lasting, taking into account that pouchitis may appear as late as 10 years after surgery. ${ }^{27} 28$ In the case of chronic continuous pouchitis, an event occurring in only $5 \%$ of the total IPAA, ${ }^{28}$ the great number of patients to be included would be an additional difficulty.

In conclusion, ANCA persist in a high proportion of UC patients after colectomy and its presence is not related to the type of surgery performed. The lower percentage found in operated patients suggests that ANCA positivity might be related to the presence of either inflamed or diseased tissue. On the other hand, ANCA positivity in UC should not be used as a marker for predicting the development of pouchitis and, at present, it does not seem a good parameter for deciding the type of surgery to be performed in UC patients.

Part of this study has been presented as a poster at the 95th Annual Meeting of the American Gastroenterological Association held in San Diego in May 1995, and published as an abstract in Gastroenterology 1995; 108: A816. 
1 Gross WL, Schmitt WH, Csernok E. ANCA and associated diseases: immunodiagnostic and pathogenic aspects. Clin Exp Immunol 1993; 91: 1-12.

2 Shanahan F. Neutrophil autoantibodies in inflammatory bowel disease: are they important? Gastroenterology 1994; 107: 586-9.

3 Gross WL, Hauschild S, Mistry N. The clinical relevance of ANCA in vasculitis. Clin Exp Immunol 1993; 93 (suppl 1): 7-11.

4 Patel RT, Stokes R, Birch D, Ibbotson J, Keighley MRB. Influence of total colectomy on serum antineutrophil cytoplasmic antibodies in inflammatory bowel disease. $\mathrm{Br}$ cytoplasmic antibodies in
J Surg 1994; 81: 724-6.

5 Lamproye A; Belaiche J, Louis E, Salmon J, Mahieu PH. Anticorps anticytoplasme des polynucléaires neutrophiles (ANCA) dans les maladies inflammatoires du tube digestif. Acta Gastroenterol Belg 1994; LVII: 171-6.

6 Broekroelofs J, Mulder AHL, Nelis GF, Westerveld BD, Cohen Tervaert JW, Kallenberg CGM. Anti-neutrophil cytoplasmic antibodies (ANCA) in sera from patients with inflammatory bowel disease (IBD). Relation to disease pattern and disease activity. Dig Dis Sci 1994; 39: 545-9.

7 Sandborn WJ, Landers CJ, Tremaine WJ, Targan SR. Antineutrophil cytoplasmic antibody correlates with chronic pouchitis after ileal pouch-anal anastomosis. $A m$ f Gastroenterol 1995; 90: 740-7.

8 Sung JY, Chan FKL, Lawton J, Leung JCK, Liew CT, Leung NWY, et al. Anti-neutrophil cytoplasmic antibodies Leung NWY, et al. Anti-neutrophil cytoplasmic antibodies (ANCA) and inflammatory

9 Ellerbroek PM, Outkerk Pool M, Ridwan BU, Dolman $\mathrm{KM}$, von Blomberg BME, von dem Borne AEGKr, et al. Neutrophil cytoplasmic antibodies (pANCA) in ulcerative colitis. $\mathcal{F}$ Clin Pathol 1994; 47: 257-62.

10 Kaneko K, Suzuki Y, Yamashiro Y, Yabuta K. Is p-ANCA in ulcerative colitis directed against b-glucoronidase? Lancet 1993; 341: 320.

11 Remaux D, Colombel JF, Heresbach D, Duclos B, Chaussade S, Belaiche J, et al. Antineutrophil cytoplasmic auto-antibodies in sera from patients after proctocolectomy for ulcerative colitis. Gastroenterology 1993; 104: A769.

12 Remaux D, Colombel JF, Heresbach D, Duclos B, Chaussade S, Duclos J, et al. ANCA and pouchitis in Chaussade S, Duclos J, et al. ANCA and pouchitis in ulcerative colitis after proctoco

13 Vecchi M, Gionchetti P, Bianchi MB, Belluzi A, Meucci G Campieri $\mathrm{M}$, et al. p-ANCA and development of pouchitis in ulcerative colitis patients after proctocolectomy and ileoanal pouch anastomosis. Lancet 1994; 344: 886-7

14 Aitola P, Miettinen A, Mattila A, Matikainen M, Soppi E. The effect of proctocolectomy on serum antineutrophil cytoplasmic antibodies in patients with ulcerative colitis Gastroenterology 1995; 108: A769.
15 Lennard-Jones JE. Classification of inflammatory bowel disease. Scand f Gastroenterol 1989; 24 (suppl 170): 2-6. 16 Truelove SC, Witts LJ. Cortisone in ulcerative colitis. Report on therapeutic trial. BMF 1955; 2: 1041-6.

17 Moskowitz RL, Shepherd NA, Nicholls RJ. An assessment of inflammation in the reservoir after restorative proctocolectomy with ileoanal ileal reservoir. Dig Dis Sci 1986; 1: 167-74.

18 Sandborn WJ, Tremaine WJ, Batts KP, Pemberton JH, Phillips SF. Pouchitis after ileal pouch-anal anastomosis: a pouchitis disease activity index. Mayo Clin Proc 1994; 69: 409-15.

19 Saxon A, Sanahan F, Landers C, Ganz T, Targan S. A distinct subset of antineutrophil cytoplasmic antibodies is associated with inflammatory bowel disease. $\mathcal{f}$ Allergy Clin associated with inflammatory

20 Oudkerk Pool M, Ellerbroek PM, Ridwan BU, Goldschmeding R, von Blomberg BME, Peña AS, et al. Serum antineutrophil cytoplasmic autoantibodies in inflammatory bowel disease are mainly associated with ulcerative colitis. A correlation study between perinuclear antineutrophil cytoplasmic autoantibodies and clinical parameters, medical and surgical treatment. Gut 1993; 34: 46-50.

21 Oudkerk Pool M, Roca M, Remaux D, Bouma G, Peña S, Colombel JF, et al. The value of p-ANCA as a serological marker for ulcerative colitis in different European regions. Eur 7 Gastroenterol Hepatol 1994; 6: 399-403.

22 Lee JCW, Lennard-Jones JE, Cambridge G. Antineutrophil antibodies in familial inflammatory bowel disease. antibodies in familial inflammat

23 Duerr RH, Neigut DA. Molecularly defined HLA-DR2 alleles in ulcerative colitis an antineutrophil cytoplasmic antibody-positive subgroup. Gastroenterology 1995; 108: 423-7.

24 Duerr RH, Targan SR, Landers CJ, LaRusso NF, Lindsay $\mathrm{KL}$, Wiesner RH, et al. Neutrophil cytoplasmic antibodies: a link between primary sclerosing cholangitis and ulcerative colitis. Gastroenterology 1991; 100: 1385-91.

25 Duerr RH, Targan SR, Landers CI, Sutherland LLR, Shanahan F. Anti-neutrophil cytoplasmic antibodies in ulcerative colitis. Comparison with other colitides and ulcerative colitis. Comparison with other colitides and

diarrheal illnesses. Gastroenterology 1991; 100: 1590-6.
26 Rump JA, Worner I, Roth M, Scholmerich J, Hansch M, Rump JA, Worner I, Roth M, Scholmerich J, Hansch M,
Peter HH. p-ANCA of undefined specificity in ulcerative Peter HH. p-ANCA of undefined specificity in ulcerative colitis: correlation to disease acti

27 Luukkonen $P$, Järvinen $H$, Tanskanen $M$, Kahri $A$. Pouchitis - recurrence of the inflammatory bowel disease? Gut 1994; 35: 243-6.

28 Sandborn WJ. Pouchitis following ileal pouch-anal anastomosis: definition, pathogenesis, and treatment. Gastroenterology 1994; 107: 1856-60. 